\title{
Weak Pitch Angle Scattering of few MV Rigidity Ions from Measurements of Anisotropies in the Distribution Function of Interstellar Pickup $\mathrm{H}^{+}$
}

\author{
G. Gloeckler \\ Department of Physics and IPST, University of Maryland. College Park \\ and Department of Atmospheric, Oceanic and Space Sciences, University of Michigan, Ann Arbor
}

\author{
N. A. Schwadron, L. A. Fisk \\ Department of Atmospheric, Oceanic and Space Sciences, University of Michigan, Ann Arbor
}

J. Geiss

Physikalisches Institut, Universität Bern, Bern, Switzerland

\begin{abstract}
The distribution function of interstellar pickup hydrogen is measured with the SWICS instrument on Ulysses at high heliographic latitudes, over a broad range of velocities, including those below that of the solar wind. It is found that these few MV rigidity protons exhibit a pronounced inward radial streaming in the frame of the solar wind, suggesting that neither instabilities generated by the pickup ions nor ambient turbulence is effective in scattering and isotropizing these particles. These results have implications for theories for the instabilities associated with pickup ions and for the propagation of low rigidity particles in the solar wind.
\end{abstract}

\section{Introduction}

Interstellar neutral gas is swept into the heliosphere by the motion of the Sun through the interstellar medium. Once here, it is ionized by photoionization and charge exchange, and then forced to gyrate about the solar wind magnetic field, thus forming a new particle population in the solar wind -- the interstellar pickup ions.

The behavior of the interstellar pickup ions has been a subject of interest for some years. The ions are expected to form the dominant internal pressure in the solar wind in the outer heliosphere, and thus can affect the solar wind flow [e.g., Axford, 1972; Holzer, 1977] and the structure of the termination shock, where the solar wind becomes subsonic. The ions are expected to excite interesting instabilities when they are first picked up by the solar wind [e.g., Wu and Davidson, 1972; Wu et al., 1973; Hartle and $W u, 1973 ; W u$ and Hartle, 1974; Lee and Ip, 1987], and thus could lead to the generation of high frequency power in the power spectrum of interplanetary turbulence. The interstellar ions are believed to be the source of the anomalous cosmic ray component seen at much higher energies and thus, at some location in the heliosphere, should undergo significant acceleration [Fisk et al., 1974].

The Ulysses mission at high heliographic latitudes provides a unique opportunity to study interstellar pickup ions

Copyright 1995 by the American Geophysical Union.

Paper number 95GL02480

0094-8534/95/95GL-02480\$03.00 in detail. The Solar Wind Ion Composition Spectrometer (SWICS) instrument on Ulysses has extremely low background and can measure separately the mass, charge, and energy of solar wind and interstellar ions. Enroute to Jupiter, as the intensities of most of the interstellar ions increased, it was possible to measure for the first time interstellar pickup hydrogen [Gloeckler et al., 1993] as well as nitrogen, oxygen and neon [Geiss et al., 1994]. At high heliographic latitudes, where, in the current solar minimum, conditions in the solar wind are quite steady, observations from SWICS can be integrated over long periods to provide detailed information on the distribution function of interstellar pickup ions. Moreover, at high latitudes, where the solar wind speed is high, the distribution function of these pickup ions with speeds below that of the solar wind is revealed.

In this paper, we present measurements of the distribution function of interstellar hydrogen at high heliographic latitudes over the full range of velocities, from below that of the solar wind to several times that of the solar wind. We find no evidence of any significant acceleration beyond that which occurs when the particles are first picked up by the solar wind. However, we find that the interstellar hydrogen ions exhibit a pronounced radial streaming towards the sun in the frame of the solar wind, with a speed several times the local Alfven speed. Although the distribution function appears to be gyrotropic about the magnetic field, and is consistent with having been spread in pitch angle, there is no evidence of the excitation of streaming instabilities which will dampen the inward streaming. The observed anisotropy can be interpreted in terms of an effective mean free path for the pickup protons which is of order $1 \mathrm{AU}$. This places a limit on the scattering which is produced by ambient turbulence, which, in turn, imposes interesting constraints on theoretical models for the scattering of low rigidity particles.

\section{Observations}

The results reported here are based on data obtained with the SWICS instrument [Gloeckler et al., 1992] on Ulysses. SWICS measures the intensity of solar wind and suprathermal ions as a function of their energy per charge $(E)$, mass $(m)$, and charge state (q), from 0.6 to $60 \mathrm{keV} / \mathrm{e}$ in logarithmically spaced steps with $\Delta \mathrm{E} / \mathrm{E} \approx 0.04$. SWICS uses techniques of energy per charge analysis, followed by post acceleration of ions by $23 \mathrm{kV}$ and a 
time-of-flight and energy measurement, to identify ions and sample their distribution functions once every 13 minutes. Because of the double and triple coincidence techniques used, the background levels are exceedingly low allowing measurements of the very low flux levels of interstellar pickup ions. The viewing direction of SWICS is such that in one spin period of Ulysses $(\sim 12 \mathrm{sec})$ a $\pi$ steradian cone, centered within $\sim 20^{\circ}$ of the solar wind direction, is sampled.

The solar wind and the pickup ions were observed with SWICS for a 30 day interval from September 1 to 30, 1994, near solar minimum conditions. During this period, Ulysses was near its highest solar latitudes, over the southern solar pole. It was thus in a stable high speed solar wind stream, with nearly constant speed of $780 \mathrm{~km} / \mathrm{s}$. These stable conditions, combined with the low background of the SWICS instrument, make it possible to integrate over the 30-day interval and obtain a distribution function for the solar wind and the interstellar hydrogen with a range of nine orders of magnitude.

Shown in Figure 1 is the observed distribution function for solar wind and interstellar pickup protons. These measurements are made in the frame of the spacecraft, and thus the interstellar ions, when they are picked up by the solar wind, acquire a velocity of up to twice that of the solar wind. Two features are evident in these observations. First, in the high speed wind, the solar wind peak is located sufficiently high in velocity to reveal the pickup ion distribution at speeds less than that of the solar wind, that is, interstellar ions that are propagating in a direction towards the Sun. Second, there is no evidence for significant densities of protons at speeds more than twice that of the solar wind, implying that in this stable solar wind stream there is little, if any, acceleration of the interstellar ions subsequent to that experienced in the pickup process. These observations should be contrasted with the acceleration of the pickup ions in Corotating Interaction Regions at lower heliographic latitudes [Gloeckler et al., 1994].

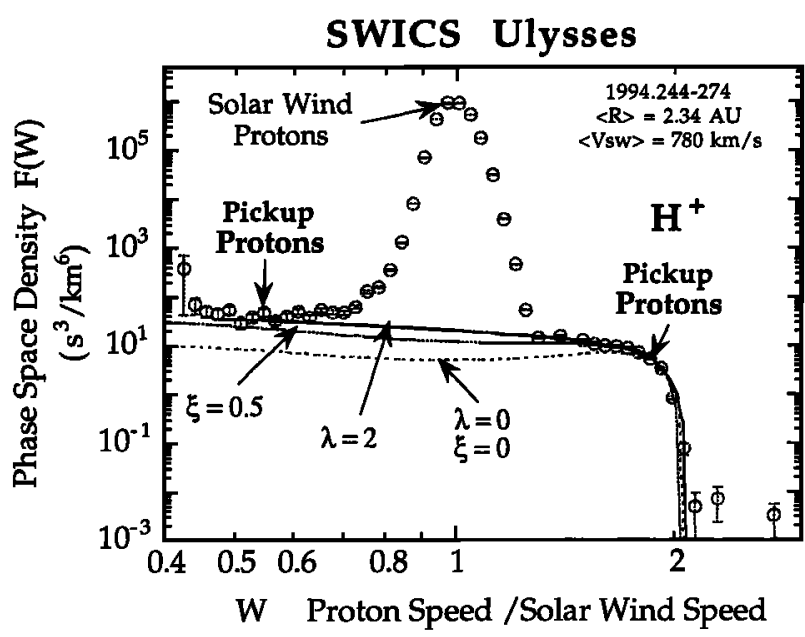

Figure 1. Phase space density of interstellar pickup protons and solar wind protons as a function of $W$ (the proton speed in the spacecraft frame divided by the solar wind speed) measured with SWICS during a one month interval in the high speed solar wind. An isotropic distribution function in the solar wind frame (curve labeled $\lambda=0, \xi=0$ ) is incompatible with the measured velocity distribution (circles). Large anisotropies $(\xi>0.5)$ and a scattering mean free path, $\lambda$, of the order of one AU are required to fit the observations.

\section{Discussion}

Vasyliunas and Siscoe [1976] obtained an analytic description for the pickup ion velocity distribution in which it was assumed that there was injection due to ionization, convection with the solar wind, and resulting adiabatic expansion. The pickup ions were assumed to be isotropic in the frame of the solar wind, and thus no anisotropic propagation was permitted. This solution for the distribution function, in the frame of the solar wind, can be written as:

$$
f_{V S}(r, \theta, w)=\frac{3}{8 \pi V^{4}} \frac{\beta_{p} r_{e}^{2} w^{-\frac{3}{2}}}{r} n_{n}\left(r w^{\frac{3}{2}}, \theta\right), \quad w \leq 1
$$

and $f_{V S}=0$ for $w>1$. Here, $r$ is the heliocentric radial distance; $\theta$ is the angle between the solar wind velocity and the upstream direction of interstellar neutrals; $\beta_{p}$ is the production rate of interstellar ions at the reference distance of $1 \mathrm{AU}$; and $w$ is the velocity of interstellar ions in the solar wind frame, in units of the solar wind speed $V$. The density of interstellar neutrals at $r$ and $\theta$ is given by $n_{n}$.

The distribution function in the frame of the solar wind can then be transformed into the frame of the spacecraft, as would be seen by the SWICS instrument. The curve marked $\lambda=0$ in Figure 1 is the transform of the Vasyliunas and Siscoe solution for interstellar pickup hydrogen for the parameters appropriate for the SWICS observations. The solar wind speed is 780 $\mathrm{km} / \mathrm{s}$, and the average heliocentric radial distance is $2.34 \mathrm{AU}$. The production rate of pickup protons was taken to be $\beta_{p}=$ $2.6 \cdot 10^{-7} \mathrm{~s}^{-1}$ in order to fit the measured phase space density at around $W=2$. For simplicity we have assumed the cold neutral model of Thomas [1978] for the interstellar neutral density, with the following parameters: the neutral wind makes a flow angle of $86^{\circ}$ with the heliocentric radial direction; the ratio of the force of radiation pressure to the gravitation force is 0.65 ; interstellar neutrals have a bulk speed of $20 \mathrm{~km} / \mathrm{s}$. The loss rate of interstellar hydrogen at $1 \mathrm{AU}$ was taken as $6 \cdot 10^{-7} \mathrm{~s}^{-1}$ and the neutral hydrogen number density outside the heliosphere as $0.08 \mathrm{~cm}^{-3}$ [Gloeckler et al., 1993].

Clearly, the curve marked $\lambda=0$ in Figure 1 falls markedly below the observations, especially for pickup ion velocities that are less than the solar wind speed in the frame of the solar wind, i.e. for pickup ions that are propagating radially inward. Using the hot temperature model [e.g., Thomas, 1978] and assuming an $8000 \mathrm{~K}$ gas and slightly different loss and production rates, results in a distribution that is indistinguishable from the $\lambda=0$ curve in the figure.

These observations can be interpreted in terms of an inward radial anisotropy $\xi$, that is, that the distribution function in the frame of the solar wind is given by

$$
f(r, \theta, \Theta, w)=f_{V S}(r, \theta, w)[1+\xi \cos (\Theta)]
$$

where $\Theta$ is the angle between the velocity of the pickup ions and the inward heliocentric radial direction. For simplicity, we assume that $\xi$ is a constant, independent of $w$. The curve marked $\xi=0.5$ in Figure 1 is the transformation of (2) into the frame of the spacecraft, with $\xi=0.5$. Using the same loss rate as before $\left(6 \cdot 10^{-7} \mathrm{~s}^{-1)}\right.$, the production rate $\beta_{p}$ of $4.5 \cdot 10^{-7} \mathrm{~s}^{-1}$ was now used to fit the data at $\mathrm{W}$ around 2 . Evidently an inward anisotropy greater than $50 \%$ is required to match the observed distribution function.

This strong anisotropy is driven by the large radial gradients of the interstellar pickup hydrogen, which results from the 
fact that interstellar neutral hydrogen penetrates easily only to within 4-5 AU of the Sun [e.g., Axford, 1972]. At the radial distance of $\approx 2.5 \mathrm{AU}$ the pickup hydrogen density is growing exponentially with radial distance [Gloeckler and Geiss, 1995]. This situation should be contrasted with interstellar neutral helium, which penetrates easily to within $1 \mathrm{AU}$ of the Sun, and thus yields small radial gradients for pickup helium. The statistics for pickup helium observed at velocities less than that of the solar wind are not sufficient, even with the long integration times available over the solar poles and with the low background of SWICS, to give an accurate determination of the helium anisotropy. However, it appears to be small, as would be expected for the small radial gradient.

An anisotropy of $50 \%$ for interstellar pickup hydrogen in the frame of the solar wind corresponds to a mean speed inward in radial distance of $\langle\nu\rangle=\xi V / 3$, where $V$ is the solar wind speed. Thus, with $V \sim 780 \mathrm{~km} / \mathrm{s},\langle v\rangle \sim 130 \mathrm{~km} / \mathrm{s}$, which is several times the Alfvén speed at the radial distance of the SWICS observations. Several authors have calculated the hydromagnetic instabilities that can be excited when the pickup ions stream relative to the mean magnetic field at greater than the Alfvén speed [e.g., Lee and Ip, 1987 and references therein], and find growth times, and thus the relaxation time of the streaming, which are small compared to the convection time of the pickup ions. Clearly, there is no evidence in the observations presented here that such instabilities are being excited.

Our measurements can also be interpreted in terms of a diffusive mean free path. Clearly, with anisotropies as large as $50 \%$, the application of diffusion theory is questionable. Nonetheless, a diffusive approach should give a valid indication of the order of magnitude of the mean free path. Further, since the speeds of the pickup ions in the solar wind frame are less than or equal to the solar wind speed, the pickup ions do not actually execute any inward radial propagation due to diffusion. However, the diffusive calculations can be performed with the distribution function measured in the frame of the solar wind, that is, with the velocities of the pickup ions measured in this frame. There is an inward radial streaming in this frame which affects the transport of the particles and thus the evolution of the distribution function.

The results of Vasyliunas and Siscoe [1976] can be extended to include the effects of particle diffusion. The behavior of the isotropic portion of the distribution function is governed by the standard steady-state transport equation [Parker, 1965], which includes convection, adiabatic deceleration, and diffusion, with a source term which represents the ionization of the neutrals and assumes that pickup ions initially form an isotropic ring distribution. However, unlike the counterpart equation for higher energy particles, in this case, where the convection speed of the solar wind and the particle speeds are comparable, it is appropriate to measure the speed or energy of the particles in the frame of the solar wind. The mean free path $\lambda$ in the radial direction is related to the mean free path along the magnetic field, $\lambda^{\prime}$, by $\lambda=\lambda^{\prime} \cos ^{2}(\Psi)$, where $\Psi$ is the angle between the radial direction and the average magnetic field direction. For tractability, we take $\lambda$ to be constant. After some calculations, the isotropic portion of the distribution function in the frame of the solar wind is found to be for $w \leq 1$,

$$
\begin{gathered}
f_{o}(r, \theta, w)=\frac{15 r_{o}}{8 \pi V^{4}} \frac{r_{o}}{\lambda} \frac{\beta_{p}}{z^{1 / 2} w^{3 / 4}} \frac{1}{1-w^{5 / 2}} \times \\
\int_{0}^{\infty} \frac{d \bar{z}}{\tilde{z}^{1 / 2}} n_{n}(\tilde{z}, \theta) \exp \left(-\frac{5 r_{o}}{\lambda} \frac{z w^{3 / 2}+\tilde{z}}{1-w^{5 / 2}}\right) I_{1}\left(\frac{10 r_{o}}{\lambda} \frac{(z \tilde{z})^{1 / 2} w^{3 / 4}}{1-w^{5 / 2}}\right)
\end{gathered}
$$

and $f_{o}=0$ for $w>1$. Again, $w$ is the ratio of the speed of the pickup ions to the solar wind speed. Here, $z$ is $r / r_{o}$ where $r$ is the heliocentric radial distance and $r_{o}$ is $1 \mathrm{AU} ; \beta_{p}$ is the production rate of pickup ions referenced to $1 \mathrm{AU} ; n_{n}$ is the density of neutral particles at $z$ and $\theta$, the angle between the solar wind velocity and the upstream direction of interstellar neutrals; and $I_{1}$ is a modified Bessel function. Note that the limits of the integral are from 0 to $\infty$, whereas, physically, particles at $\tilde{z}>z$ cannot contribute. However, for values of the parameters used here, the contribution from $\tilde{z}>z$ is small and negligible. In the case $\lambda=0$, (3) reduces to (1).

The full distribution function, including the effects of diffusion, is then given by

$$
f(r, \theta, \Theta, w)=f_{o}(r, \theta, w)-\lambda \cos (\Theta) \frac{\partial f_{o}}{\partial r}
$$

The resulting anisotropy is driven by the gradient of pickup ions, which in the case of protons is positive and large. There can also be a residual anisotropy in the case of long mean free path that results from the initial streaming of the particles along field lines immediately following their ionization, as has been observed for pickup helium at 1 AU (E. Möbius, personal communication, 1995). Equation (4), then, should understate the actual anisotropy. Using the same parameters as in the evaluation of (1), except for the production rate which is taken to be $\beta_{p}=4 \cdot 10^{-7} \mathrm{~s}^{-1}$ to match the observations at around $W=2$, and with the Thomas [1978] cold neutral model for the interstellar neutral density with the parameters listed above, (4) was evaluated for various values of $\lambda$ and transformed into the frame of the spacecraft. The results are shown in Figure 1 for $\lambda=2 \mathrm{AU}$, which provides the best fit to the observations. It would be unfair, given the limitations of diffusion theory in this case of large anisotropies, to use this approach to specify an exact value for the mean free path. Nonetheless, it is of order $1 \mathrm{AU}$.

Pickup hydrogen ions are of course very low rigidity $(\leq 2.4$ MV) particles. With this inferred mean free path, then we have, for the first time, an indication of the mean free path for very low rigidity ions, which places interesting constraints on the scattering due to ambient turbulence of low rigidity particles and on the theories which describe this scattering. Palmer [1982] has compiled observations of the mean free paths for energetic particles in the solar wind over a wide range of particle rigidities (the "Palmer consensus"), and demonstrated that quasi-linear models of particle interaction with slab turbulence [e.g., Jokipii, 1966] fail to account for the large mean free paths observed for low rigidity particles. However, Bieber et al. [1994] have pointed out that the large mean free path at low rigidities is observed for electrons; ions are observed at much higher rigidities than electrons. Hence, they argued that the mean free path for electrons and ions might be fundamentally different. The observations presented here clearly contradict this possibility. The rigidity of the pickup protons is $\leq 2.4 \mathrm{MV}$, which is comparable to that of low energy electrons. The mean free path inferred here for low rigidity ions of approximately $1 \mathrm{AU}$ thus falls in the range observed for comparable rigidity electrons deduced mostly from measurements at one $\mathrm{AU}$.

\section{Conclusions}

We have used the SWICS instrument on Ulysses to observe the distribution function of interstellar pickup hydrogen at high heliographic latitudes over a broad range in velocities, 
including those below that of the solar wind. We find that the interstellar hydrogen ions exhibit a strong inward radial streaming, with an anisotropy of order $50 \%$ in the frame of the solar wind and an effective mean free path for scattering of order $1 \mathrm{AU}$. These observations suggest that (i) the in[Fisk et al., 1974] stabilities that could be generated by the streaming of the pickup ions along the magnetic field are not being readily excited; and (ii) the scattering of the pickup ions by ambient turbulence yields a mean free path of order $1 \mathrm{AU}$ that falls in the range deduced for comparable rigidity energetic electrons. This long mean free path remains a challenge to account for by current theories of particle scattering in the solar wind.

Acknowledgments. We are extremely grateful to the many individuals at the University of Maryland, the University of Bern, the Max-PlanckInstitut für Aeronomie and the Technical University of Braunschweig who have developed, fabricated, and calibrated the SWICS instrument and implemented the software to reduce the data. This work was supported by NASA/JPL contract 955460 and the Swiss National Science Foundation.

\section{References}

Axford, W. I., The interaction of the solar wind with the interstellar medium, Solar Wind, NASA Spec. Publ. 308, 609, 1972.

Bieber, J. W., W. H. Matthaeus, and C. W. Smith, Proton and electron mean free paths: the palmer consensus revisited, Astrophys. J. 420, 294, 1994.

Fisk, L. A., B. Kozlovsky, and R. Ramaty, An interpretation of the observed oxygen and nitrogen enhancements in low-energy cosmic rays, Astrophys. J., 190, L35, 1974.

Geiss, J., G. Gloeckler, U. Mall, R. von Steiger, A. B. Galvin, and K. W. Ogilvie, Interstellar oxygen, nitrogen, and neon in the heliosphere, Astron. Astrophys., 282, 924, 1994.

Gloeckler, G., and J. Geiss, Observations of interstellar pickup ions, Cosmic Winds and the Heliosphere, edited by J. R. Jokipii, C. P. Sonnett, and M. S. Giampapa, University of Arizona Press, in press, 1995.

Gloeckler, G., J. Geiss, H. Balsiger, P. Bedini, J. C. Cain, J. Fischer, L. A. Fisk, A. B. Galvin, F. Gliem, D. C. Hamilton, J. V. Hollweg, F. M. Ipavich, R. Joss, S. Livi, R. Lundgren, U. Mall, J. F. McKenzie, K. W. Ogilvie, F. Ottens, W. Rieck, E. O. Tums, R. von Steiger, W. Weiss and B. Wilken, The solar wind ion composition spectrometer, Astron. Astrophys. Suppl. Ser. 92, 267, 1992.

Gloeckler, G., J. Geiss, H. Balsiger, L. A. Fisk, A. B. Galvin, F. M. Ipavich, K. W. Ogilvie, R. von Steiger, and B. Wilken, Detection of interstellar pickup hydrogen in the solar system, Science, 261,70 , 1993.

Gloeckler, G., J. Geiss, E. C. Roelof, L. A. Fisk, F. M. Ipavich, K. W. Ogilvie, L. J. Lanzerotti, R. von Steiger, and B. Wilken, Acceleration of interstellar pickup ions in the disturbed solar wind observed on Ulysses, J. Geophys. Res., 99, 17637, 1994.

Hartle, R. E., and C. S. Wu, Effects of electrostatic instabilities on planetary and interstellar ions in the solar wind, J. Geophys. Res., 78, 5802, 1973.

Holzer, T. E., Neutral hydrogen in interplanetary space, Rev. Geophys. Space Phys., 15, 467, 1977.

Jokipii, J. R., Cosmic ray propagation, 1, charged particles in a random magnetic field, Astrophys. J. 146, 480, 1966.

Lee, M. A., and W.-H. Ip, Hydromagnetic wave excitation by ionized interstellar hydrogen and helium in the solar wind, J. Geophys. Res., 92, $11041,1987$.

Palmer, I. D., Transport coefficients of low-energy cosmic rays in interplanetary space, Rev. Geophys. Space Phys., 20, 335, 1982.

Parker, E. N., The passage of energetic charged particles through interplanetary space, Planet. Space Sci., 13, 9, 1965.

Thomas, G. E., The interstellar wind and its influence on the interplanetary environment, Ann. Rev. Earth Planet. Sci., 6, 173, 1978.

Vasyliunas, V. M., and G. L. Siscoe, On the flux and energy spectrum of interstellar ions in the solar system, J. Geophys. Res., 81, 1247, 1976.

Wu, C. S., and R. C. Davidson, Electromagnetic instabilities produced by neutral particle ionization in interplanetary space, J. Geophys. Res., $77,5399,1972$.

Wu, C. S., and R. E. Hartle, Further remarks on plasma instabilities produced by ions born in the solar wind, J. Geophys. Res., 79, 283 , 1974.

Wu, C. S., R. E. Hartle, and K. W. Ogilvie, Interaction of singly charged interstellar helium ions with the solar wind, J. Geophys. Res., 78, 306, 1973.

G. Gloeckler, Department of Physics, University of Maryland, College Park, MD 20742 (e-mail: gloeckler@umdsp.umd.edu)

N. A. Schwadron, Department of Atmospheric, Oceanic and Space Sciences, Univeristy of Michigan, Ann Arbor, MI 48109 (e-mail: nathanas@engin.umich.edu)

L. A. Fisk, Department of Atmospheric, Oceanic and Space Sciences, University of Michigan, Ann Arbor, MI 48109 (e-mail: lafisk@umich.edu)

J. Geiss, Physikalisches Institut, Universität Bern, Sidlerstr. 5, CH3102 Bern, Switzerland (e-mail: geiss@phim.unibe.ch)

(Received June 13, 1995; revised July 28, 1995; accepted August 8, 1995) 